\title{
Desfechos da eletroestimulação do nervo tibial posterior no tratamento da bexiga neurogênica
}

\author{
Outcomes of electric stimulation of the posterior tibial nerve \\ in the treatment of neurogenic bladder
}

\author{
Resultados de la electroestimulación del nervio tibial \\ posterior en el tratamiento de la vejiga neurogénica
}

\author{
Railson Carlos Olinto de Brito ${ }^{1}$, Vinicius Batista Lima², \\ Maria do Carmo Pinto Lima ${ }^{3}$
}

1.Graduando em Fisioterapia na Universidade Estadual da Paraíba (UEPB), departamento de Fisioterapia. Campina Grande-PB, Brasil. https://orcid.org/0000-0001-6844-2831

2.Graduando em Fisioterapia na Universidade Estadual da Paraíba (UEPB), departamento de Fisioterapia. Campina Grande-PB, Brasil. https://orcid.org/0000-0001-7726-3712

3.Doutora em saúde materno infantil pelo Instituto de Medicina Integral Prof. Fernando Figueira (IMIP). Recife-PE, Brasil. https://orcid.org/0000-0001-5414-3582

\begin{abstract}
Resumo
Objetivo. Investigar os desfechos clínicos da eletroestimulação do nervo tibial posterior (ENTP) no tratamento da bexiga neurogênica (BN) em pacientes com diversos distúrbios neurológicos. Método. Foi realizada uma revisão de integrativa de ensaios clínicos publicados nos últimos cinco anos, nas bases de dados PubMed, Embase, Cochrane library e ScienceDirect. Os descritores usados foram: "Urinary bladder", "Neurogenic", "Electric stimulation" e "Posterior tibial". Foram incluídos na revisão os ensaios clínicos com pacientes exclusivamente neurológicos, com sintomas de BN, conscientes, que realizaram ingestão de água contínua e sem contraindicações para a eletroestimulação. Foram excluídos os estudos que fizeram uso de animais, que utilizaram outras intervenções nos indivíduos submetidos à ENTP e que tiveram em sua amostra pessoas com lesões neurais periféricas graves. Resultados. Foram encontrados 287 artigos, sendo, no final da análise, selecionados apenas seis. A amostra total entre os estudos foi composta por indivíduos de ambos os sexos, portadores de três distúrbios neurológicos distintos: lesão medular, Parkinson e Esclerose múltipla. Todas as aplicações ocorreram unilateralmente, no modo corrente contínua, com parâmetros entre 10 ou $20 \mathrm{HZ}$ e largura de pulso de 200 us. Como resultado, a ENTP alcançou desfechos positivos na qualidade de vida e na diminuição de sintomas como frequência urinária, incontinência urinária, urgência urinária e noctúria. Conclusão. A ENTP demostra trazer bons desfechos clínicos a portadores de BN e sua aplicação pode ser usada, com segurança, no tratamento de pacientes com disfunções neurológicas distintas.
\end{abstract}

Unitermos. Bexiga urinária neurogênica; Estimulação elétrica; Nervo tibial posterior; Sintomas clínicos

\footnotetext{
Abstract

Objective. To investigate the clinical outcomes of electric stimulation of the posterior tibial nerve (ENTP) in the treatment of neurogenic bladder (BN) in patients with various neurological disorders. Method. An integrative review of clinical trials published in the last five years was carried out in the PubMed, Embase, Cochrane library and ScienceDirect databases. The descriptors used were: "Urinary bladder", "Neurogenic", "Electric stimulation" and "Posterior tibial". Included in the review were clinical trials with exclusively neurological patients, conscious, with BN symptoms, who performed continuous water intake and without
} 
contraindications for electrostimulation. Studies that used animals, that used other interventions in individuals submitted to ENTP and that had in their sample people with severe peripheral neural injuries were excluded. Results. 287 articles were found, and at the end of the analysis, only six were selected. The total sample between studies was composed of individuals of both sexes, with three different neurological disorders: spinal cord injury, Parkinson's and multiple sclerosis. All applications occurred unilaterally, in direct current mode, with parameters between 10 or $20 \mathrm{HZ}$ and pulse width of $200 \mu \mathrm{s}$. As a result, ENTP achieved positive outcomes in quality of life and decreased symptoms such as urinary frequency, urinary incontinence, urinary urgency and nocturia. Conclusion. ENTP demonstrates good clinical outcomes for BN patients and its application can be used safely in the treatment of patients with different neurological disorders.

Keywords. Neurogenic urinary bladder; Electrical stimulation; Posterior tibial nerve; Clinical symptoms

\begin{abstract}
Resumen
Objetivo. Investigar los resultados clínicos de la electroestimulación del nervio tibial posterior (ENTP) en el tratamiento de la vejiga neurogénica (BN) en pacientes con diversos trastornos neurológicos. Método. Se realizó una revisión integradora de los ensayos clínicos publicados en los últimos cinco años en las bases de datos PubMed, Embase, Cochrane library y ScienceDirect. Los descriptores utilizados fueron: "Vejiga urinaria", "Neurogénico", "Estimulación eléctrica" y "Tibial posterior". Se incluyeron en la revisión ensayos clínicos con pacientes exclusivamente neurológicos, conscientes, con síntomas de BN, que realizaban ingesta continua de agua y sin contraindicaciones para la electroestimulación. Se excluyeron los estudios que utilizaron animales, que utilizaron otras intervenciones en individuos sometidos a ENTP y que tenían en su muestra a personas con lesiones neurales periféricas graves. Resultados. Se encontraron 287 artículos y al final del análisis solo se seleccionaron seis. La muestra total entre los estudios estuvo compuesta por individuos de ambos os sexos, con tres trastornos neurológicos diferentes: lesión medular, Parkinson y esclerosis múltiple. Todas las aplicaciones ocurrieron de forma unilateral, en modo de corriente continua, con parámetros entre 10 o $20 \mathrm{HZ}$ y ancho de pulso de $200 \mu \mathrm{s}$. Como resultado, ENTP logró resultados positivos en la calidad de vida y en la reducción de síntomas como frecuencia urinaria, incontinencia urinaria, urgencia urinaria y nicturia. Conclusión. ENTP muestra buenos resultados clínicos para los pacientes con BN y su aplicación se puede utilizar de forma segura en el tratamiento de pacientes con diferentes trastornos neurológicos.
\end{abstract}

Palabras clave. Vejiga urinaria neurogênica; Estimulación eléctrica; Nervio tibial posterior; Síntomas clínicos

Trabalho realizado na Universidade Estadual da Paraíba (UEPB), Campina Grande-PB, Brasil.

\title{
INTRODUÇÃO
}

\author{
A bexiga hiperativa neurogênica (BN) é uma disfunção
}

\section{do trato urinário inferior causada por distúrbios neurais}

centrais e periféricos. Essa patologia afeta a sensibilidade vesical e/ou equilíbrio vesico-esfincteriano, podendo se apresentar de forma estável ou progressiva, dependendo da doença causadora ${ }^{1}$. 
O aparecimento da BN não é unânime entre pacientes neurológicos, porém é comum. Assim, doenças neurais como o Parkinson, Esclerose múltipla, lesões medulares e Acidente Vascular Cerebral (AVC), cursam com uma alta incidência de $\mathrm{BN}^{2}$.

Cerca de $80 \%$ dos pacientes que passaram por um evento de AVC, nos primeiros seis meses de recuperação, desenvolvem incontinência urinária (IU) ligada à BN. A IU é um dos sintomas mais comuns da BN, contudo, também há o desenvolvimento de complicações como infecção urinária, urgência urinária (UU), noctúria e dor miccional ${ }^{3,4}$.

Com esses problemas urológicos, há um grande impacto econômico. O aumento nas internações ligadas a infecções urinárias e as despesas associadas com intervenções cirúrgicas, geram gastos consideráveis no manejo desses pacientes ${ }^{5,6}$.

Dentre os recursos destinados ao tratamento das complicações da BN, estão os tratamentos medicamentosos e invasivos. Contudo o uso de determinadas drogas e a aplicação de procedimentos cirúrgicos em pacientes com BN, podem causar o aparecimento de efeitos colaterais complexos e até ter certa ineficiência a longo prazo ${ }^{7}$.

A eletroestimulação, entretanto, apresenta-se como um tratamento complementar viável, cuja aplicação possui bons desfechos, fácil manejo, baixo investimento e ausência de efeitos colaterais ${ }^{8,9}$. Um dos tipos de eletroestimulação mais usados é a do nervo tibial posterior (ENTP). Os seus bons resultados estão ligados à neuromodulação das interações 
sinápticas lombares e sacrais entre L4-S2. Acredita-se que essa função neuromoduladora pode estar relacionada à melhoria da atividade vesical da bexiga ${ }^{10-12}$.

Alguns estudos levaram em consideração o uso da ENTP no tratamento da BN, todavia, as revisões existentes se limitam a uma única patologia ou englobam outras disfunções na análise de seus resultados, como é o caso da bexiga idiopática. Diante do exposto, o objetivo desta revisão foi investigar os desfechos clínicos da ENTP no tratamento da BN de pacientes com diversos distúrbios neurológicos.

\section{MÉTODO}

Foi desenvolvido um estudo do tipo revisão integrativa, incluindo os ensaios clínicos publicados nos últimos cinco anos. Os artigos utilizados nesta revisão integrativa foram selecionados por meio de um levantamento de periódicos com evidência científica, feito no mês novembro de 2020. A busca foi feita no idioma inglês, nas bases de dados eletrônicas PubMed, Embase, Cochrane library e ScienceDirect. Para a construção da estratégia, foram utilizados os seguintes descritores: "Urinary Bladder", "Neurogenic", "Electric Stimulation", "Posterior Tibial".

Foram incluídos na revisão os ensaios clínicos com pacientes exclusivamente neurológicos, com sintomas de $\mathrm{BN}$, conscientes, que realizaram ingestão de água contínua e sem contraindicações à eletroestimulação. Foram excluídos os artigos que fizeram uso de animais em suas intervenções, que utilizaram outras intervenções nos indivíduos 
submetidos à ENTP e que incluíram em sua amostra pessoas com lesões neurais periféricas graves.

Foram adotadas as seis etapas indicadas para a constituição da revisão integrativa da literatura: 1) seleção da pergunta de pesquisa; 2) definição dos critérios de inclusão de estudos e seleção da amostra; 3) representação dos estudos selecionados em formato de tabelas, considerando todas as características em comum; 4) análise crítica dos achados, identificando diferenças e conflitos; 5) interpretação dos resultados; 6) reportar, de forma clara, a evidência encontrada ${ }^{13}$.

\section{RESULTADOS}

Foram identificados 287 estudos em todas as bases de dados. Após a avaliação dos textos completos e da aplicação dos critérios de inclusão e exclusão, seis artigos entraram nos padrões de elegibilidade, sendo quatro artigos excluídos pelos critérios de exclusão. A Figura 1 identifica as etapas de busca.

A amostra total entre todos os estudos foi composta por ambos os gêneros, sendo uma porcentagem de 57,2\% homens $(H), 20,1 \%$ mulheres $(M)$ e 22,5\% com gênero não informado (NI). A idade média foi de 46,5 anos. As disfunções neurológicas abordadas nos estudos foram lesão medular, doença de Parkinson ou Esclerose múltipla. 
Figura 1. Fluxograma de busca e seleção dos artigos durante o processo de revisão integrativa.

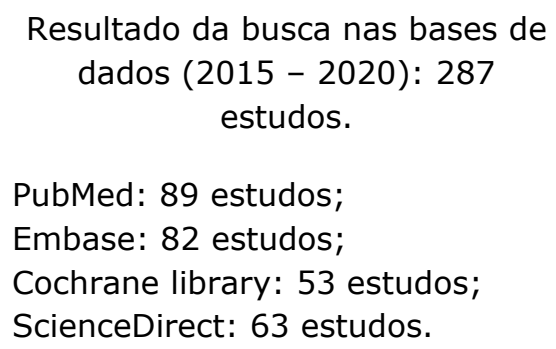

Estudos selecionados após a leitura dos títulos e resumos: 17 estudos.

PubMed: 6 estudos;

Embase: 7 estudos;

Cochrane library: 2 estudos;

ScienceDirect: 2 estudos.

Estudos selecionados após a exclusão das duplicatas: 10.

PubMed: 6 estudos;

Embase: 3 estudos;

Cochrane library: 0 estudo;

ScienceDirect: 1 estudo.

Todos os artigos optaram pela colocação unilateral dos eletrodos próximo ao maléolo medial, no modo corrente contínua e com a intensidade até flexão do dedo do pé. Três estudos submeteram os pacientes a dez sessões de ENTP, com duração de 30 minutos, frequência (FR) de $10 \mathrm{HZ}$ e largura de pulso de $200 \mu s^{9,14,15}$. Os demais utilizaram a ENTP 
em uma FR de $20 \mathrm{HZ}$ associada a uma largura de pulso de $200 \mu$ s. Além disso, optaram por oito sessões de ENTP de 30 minutos, por 12 e 48 sessões com tempo não especificado $8,16,17$.

Nesta revisão, os desfechos da ENTP foram analisados de três formas diferentes. Primeiro, foram comparados com a linha base dos sintomas de cada disfunção neurológica. Logo após, os desfechos dos grupos que receberam a ENTP (G-ENTP) foram comparados aos encontrados nos grupos controle (GC). Por fim, a evolução na qualidade de vida foi mensurada a partir da sua linha de base e comparada à encontrada nos GC.

Os pacientes com lesões medulares representaram 61,9\% da amostra total. Com aplicação da ENTP, foi visto uma melhoria média de $52,1 \%$ a partir da linha de base dos sintomas. No caso da lesão medular, os sintomas (meio de avaliação) foram: primeiro desejo miccional e primeira sensação miccional (cistometria); dor (Neuropathic Pain Scale - NPS) e IU (peso da fralda) ${ }^{14-16}$.

Os indivíduos com Parkinson corresponderam a 28,1\% de toda a amostra. Com o uso da ENTP, houve uma melhoria média de $54,3 \%$ a partir da linha de base dos sintomas. $\mathrm{Na}$ doença de Parkinson, os sintomas (meio de avaliação) foram: frequência urinária; IU; UU e noctúria (International Consultation on Incontinence Questionnaire - Short Form ICIQ-SF, Overactive Bladder Questionnaire - OAB-V8, Overactive Bladder Questionnaire Short Form - OAB-q SF e diário miccional) $)^{8,9}$. 
Os pacientes com Esclerose múltipla representaram 9,8\% da amostragem geral. Com a aplicação da ENTP, houve uma melhoria de $67,8 \%$ a partir da linha de base dos sintomas. Na esclerose múltipla, os sintomas (meio de avaliação) foram: frequência urinária; IU; UU; noctúria (ICIQ-SF, OABv8 e Overactive Bladder Questionnaire - OABq) ${ }^{17}$.

Três estudos selecionados comparam os desfechos do G-ENTP com o $\mathrm{GC}^{9,14,15}$. Juntos, obtiveram uma melhoria média de 54\% nos sintomas do G-ENTP e de 8,7\% nos do GC. Dois artigos avaliaram a evolução na qualidade de vida dos pacientes ${ }^{9,16}$. Os desfechos demostraram um avanço médio de $43,8 \%$ com relação à linha base dos sintomas. A comparação dos desfechos do G-ENTP com o GC foi feita por um único estudo ${ }^{9}$, esse observou uma evolução de 43,8\% na qualidade de vida. O Quadro 1 contém os detalhes amostrais, intervencionais e resultados clínicos.

\section{DISCUSSÃO}

A ENTP demostrou efetividade na diminuição dos sintomas da BN em lesados medulares, portadores da doença de Parkinson e portadores de Esclerose múltipla. Os mesmos resultados foram vistos na comparação com os GC e na evolução da qualidade de vida.

Os bons desfechos encontrados nessas diferentes disfunções neurológicas, mesmo na presença de fisiopatologias distintas, se devem à forma universal que a ENTP age. Quando aplicada, a corrente elétrica ascende por 


\section{meio do nervo tibial posterior e abre passagem através dos nervos simpáticos pélvicos.}

Quadro 1. Amostra, intervenção e efeitos clínicos da ENTP da revisão integrativa.

\begin{tabular}{|c|c|c|c|c|}
\hline $\begin{array}{l}\text { AUTOR } \\
\text { (ANO) }\end{array}$ & PACIENTES & INTERVENÇÃO & $\begin{array}{c}\text { RESULTADOS } \\
\text { (FORMA AVALIATIVA) }\end{array}$ & CONCLUSÃO \\
\hline $\begin{array}{c}\text { Stampas et } \\
\text { al, 201914 }\end{array}$ & $\begin{array}{l}\text { Número (gênero): } \\
\text { G-ENTP: } 10 \text { (NI) } \\
\text { GC: } 6 \text { (NI) } \\
\text { Idade média: } \\
\text { G-ENTP: } 38.1 \text { anos } \\
\text { GC: } 51.8 \text { anos } \\
\text { Patologia: } \\
\text { lesão medular, com } \\
\text { diferentes graus de } \\
\text { complicação. }\end{array}$ & $\begin{array}{l}\text { Período: } \\
\text { G-ENTP: } 10 \text { sessões } \\
\text { Parâmetros: } \\
\text { ENTP unilateral por } \\
\text { corrente contínua, por } \\
30 \text { minutos, próximo ao } \\
\text { maléolo medial. FR de } \\
10 \text { Hz e largura de } \\
\text { pulso de } 200 \text { } \mu \text { s. } \\
\text { Intensidade até flexão } \\
\text { do dedo do pé. } \\
\text { GC: } 10 \text { sessões de } \\
\text { ENTP, sem corrente } \\
\text { elétrica. }\end{array}$ & $\begin{array}{l}\text { G-ENTP: aumento no } \\
\text { limiar do primeiro desejo } \\
\text { miccional em } 10 \% \text {. Sem } \\
\text { alterações na primeira } \\
\text { sensação miccional. } \\
\text { (cistometria). } \\
\text { GC: sem alterações na } \\
\text { primeira sensação } \\
\text { miccional e desejo } \\
\text { miccional (cistometria). }\end{array}$ & $\begin{array}{l}\text { A ENTP } \\
\text { demostra ser } \\
\text { ligeiramente } \\
\text { superior ao GC, } \\
\text { no tratamento } \\
\text { dos sintomas da } \\
\text { BN em lesados } \\
\text { medulares. }\end{array}$ \\
\hline $\begin{array}{l}\text { Kabay et al, } \\
2016^{8}\end{array}$ & $\begin{array}{l}\text { Número (gênero): } \\
26(\mathrm{H}) / 21(\mathrm{M}) \\
\text { Idade média: } \\
61 \text { anos } \\
\text { Patologia: } \\
\text { Parkinson, com } \\
\text { diferentes graus de } \\
\text { complicações. }\end{array}$ & 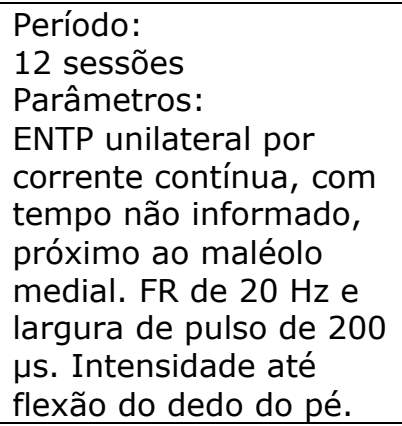 & $\begin{array}{l}\text { Diminuição da frequência } \\
\text { urinária em 40,1\%, da } \\
\text { UU em } 68 \% \text {, da IU em } \\
61,3 \% \text { e da noctúria em } \\
60,9 \% \text { (ICIQ-SF, } \\
\text { OABv8 e OAB-q). }\end{array}$ & $\begin{array}{l}\text { A ENTP } \\
\text { demostra } \\
\text { melhorar os } \\
\text { sintomas do } \\
\text { trato urinário } \\
\text { inferior em } \\
\text { pacientes com } \\
\text { doença de } \\
\text { Parkinson com } \\
\text { BN. }\end{array}$ \\
\hline $\begin{array}{l}\text { Stampas et } \\
\text { al, } 2018^{15}\end{array}$ & $\begin{array}{l}\text { Número (gênero): } \\
\text { G-ENTP: } 12 \text { (NI) } \\
\text { GC: } 7 \text { (NI) } \\
\text { Idade média: } \\
\text { G-ENTP: } 36.3 \text { anos } \\
\text { GC: } 48.3 \text { anos } \\
\text { Patologia: } \\
\text { lesão medular, com } \\
\text { diferentes graus de } \\
\text { complicação. }\end{array}$ & $\begin{array}{l}\text { Período: } \\
\text { G-ENTP: } 10 \text { sessões } \\
\text { Parâmetros: } \\
\text { ENTP unilateral por } \\
\text { corrente contínua, por } \\
30 \text { minutos, próximo ao } \\
\text { maléolo medial. FR de } \\
10 \text { Hz e largura de } \\
\text { pulso de } 200 \mu \text { s. } \\
\text { Intensidade até flexão } \\
\text { do dedo do pé. } \\
\text { GC: } 10 \text { sessões de } \\
\text { ENTP, sem corrente } \\
\text { elétrica. }\end{array}$ & $\begin{array}{l}\text { G-ENTP: aumento do } \\
\text { limiar do primeiro desejo } \\
\text { miccional em } 85,5 \% \text {. } \\
\text { Melhoria da primeira } \\
\text { sensação miccional em } \\
79,5 \% \text { (cistometria). } \\
\text { Diminuição da dor em } \\
56,5 \% \text { (NPS). } \\
\text { GC: Aumento do limiar } \\
\text { do primeiro desejo } \\
\text { miccional em } 7,5 \% \\
\text { (cistometria). Sem } \\
\text { alterações nos } \\
\text { parâmetros de primeira } \\
\text { sensação miccional e dor } \\
\text { (cistometria e NPS). }\end{array}$ & $\begin{array}{l}\text { A ENTP é uma } \\
\text { modalidade } \\
\text { segura e viável, } \\
\text { e pode ser } \\
\text { utilizada na } \\
\text { reabilitação } \\
\text { hospitalar de } \\
\text { pacientes com } \\
\text { lesão medular } \\
\text { com BN. }\end{array}$ \\
\hline
\end{tabular}


Quadro 1. Amostra, intervenção e efeitos clínicos da ENTP da revisão integrativa.

\begin{tabular}{|c|c|c|c|c|}
\hline $\begin{array}{l}\text { AUTOR } \\
\text { (ANO) }\end{array}$ & PACIENTES & INTERVENÇÃO & $\begin{array}{c}\text { RESULTADOS } \\
\text { (FORMA AVALIATIVA) }\end{array}$ & CONCLUSÃO \\
\hline $\begin{array}{l}\text { Chen; Liao; } \\
\text { Li, 201516 }\end{array}$ & $\begin{array}{l}\text { Número (gênero): } \\
\text { G-ENTP: } 46(\mathrm{H}) / 3 \\
\text { (M) } \\
\text { G-Succinato de } \\
\text { Solifenacina: } 45(\mathrm{H}) \text { / } \\
3 \text { (M) } \\
\text { Idade média: } \\
\text { G-ENTP: } 32.9 \text { anos } \\
\text { G-Succinato } \\
\text { Solifenacina: } 33.5 \\
\text { anos } \\
\text { Patologia: } \\
\text { lesão medular, com } \\
\text { diferentes graus de } \\
\text { complicações. }\end{array}$ & $\begin{array}{l}\text { Período: } \\
\text { G-ENT: } 8 \text { sessões } \\
\text { Parâmetros: } \\
\text { ENTP unilateral por } \\
\text { corrente contínua, por } \\
30 \text { minutos, próximo ao } \\
\text { maléolo medial. FR de } \\
20 \text { Hz, corrente } \\
\text { contínua, largura de } \\
\text { pulso de } 200 \text { } \mu \text { s. } \\
\text { Intensidade até flexão } \\
\text { do dedo do pé. } \\
\text { G-Succinato de } \\
\text { Solifenacina: } 5 \text { mg por } \\
\text { dia de Succinato de } \\
\text { Solifenacina, sem } \\
\text { ENTP. }\end{array}$ & $\begin{array}{l}\text { G-ENTP: diminuição da } \\
\text { IU em } 29,3 \% \text {. Aumento } \\
\text { da qualidade de vida em } \\
62,3 \% \text { (peso da fralda e } \\
\text { I-QOI). } \\
\text { G-Succinato } \\
\text { Solifenacina: diminuição } \\
\text { da IU em 40,4 \%. } \\
\text { Aumento da qualidade } \\
\text { de vida em } 64 \% \text {. Alguns } \\
\text { pacientes apresentaram } \\
\text { boca seca (peso da } \\
\text { fralda e I-QOI). }\end{array}$ & $\begin{array}{l}\text { A ENTP } \\
\text { demostra ser } \\
\text { um tratamento } \\
\text { eficaz para BN } \\
\text { de indivíduos } \\
\text { com lesão } \\
\text { medular, e } \\
\text { pode ser } \\
\text { aplicada no } \\
\text { tratamento } \\
\text { desses } \\
\text { pacientes. }\end{array}$ \\
\hline $\begin{array}{l}\text { Perissinotto } \\
\text { et al, } 2015^{9}\end{array}$ & $\begin{array}{l}\text { Número (gênero): } \\
\text { G-ENTP: } 8 \text { (NI) / GC: } \\
5 \text { (NI) } \\
\text { Idade média: } \\
\text { GC: } 63.5 \text { anos / GC: } \\
57 \text { anos } \\
\text { Patologia: } \\
\text { Parkinson, com } \\
\text { diferentes graus de } \\
\text { complicações. }\end{array}$ & $\begin{array}{l}\text { Período: } \\
10 \text { sessões } \\
\text { Parâmetros: } \\
\text { ENTP unilateral por } \\
\text { corrente contínua, por } \\
30 \text { minutos, próximo ao } \\
\text { maléolo medial. FR de } \\
10 \mathrm{~Hz} \text { e largura de } \\
\text { pulso de } 200 \mu \text { s. } \\
\text { Intensidade até flexão } \\
\text { do dedo do pé. } \\
\text { GC: } 10 \text { sessões de } \\
\text { ENTP, sem corrente } \\
\text { elétrica. }\end{array}$ & $\begin{array}{l}\text { G-ENTP: Diminuição da } \\
\text { frequência urinária em } \\
66,6 \% \text {, da IU em } 33,3 \\
\% \text { e da noctúria em } \\
50 \% \text {. Aumento da } \\
\text { qualidade de vida em } \\
43,8 \% \text {. (Relato do } \\
\text { número de eventos, ICIQ } \\
\text { - SF e OAB-V8). } \\
\text { GC: Diminuição da } \\
\text { noctúria em } 10 \% \text {. Sem } \\
\text { alterações nos } \\
\text { parâmetros de } \\
\text { frequência urinária, IU e } \\
\text { qualidade de vida } \\
\text { (Relato do número de } \\
\text { eventos, ICIQ - SF e } \\
\text { OAB-V8). }\end{array}$ & $\begin{array}{l}\text { Os resultados } \\
\text { sugerem que a } \\
\text { ENTP é eficaz } \\
\text { no tratamento } \\
\text { dos sintomas do } \\
\text { trato urinário } \\
\text { inferior de } \\
\text { indivíduos com } \\
\text { doença de } \\
\text { Parkinson. }\end{array}$ \\
\hline $\begin{array}{l}\text { Kabay et al, } \\
201717\end{array}$ & $\begin{array}{l}\text { Número (gênero): } \\
5(\mathrm{H}) / 16(\mathrm{M}) \\
\text { Idade média: } \\
42.7 \text { anos } \\
\text { Patologia: } \\
\text { Esclerose múltipla, } \\
\text { com diferentes graus } \\
\text { de complicações. }\end{array}$ & $\begin{array}{l}\text { Período: } \\
48 \text { sessões ( } 12 \text { sessões } \\
\text { a cada } 3 \text { meses) } \\
\text { Parâmetros: } \\
\text { ENTP unilateral por } \\
\text { corrente contínua, com } \\
\text { tempo não informado, } \\
\text { próximo ao maléolo } \\
\text { medial. FR de } 20 \mathrm{~Hz} \text { e } \\
\text { largura de pulso de } 200 \\
\mu \text { s. Intensidade até } \\
\text { flexão do dedo do pé. }\end{array}$ & $\begin{array}{l}\text { Diminuição da frequência } \\
\text { urinária em } 41,5 \% \text {, da } \\
\text { UU em } 78,8 \% \text {, da IU em } \\
80,2 \% \text { e da noctúria em } \\
70,7 \% \text { (ICIQ-SF, OABv8 } \\
\text { e OAB-q). }\end{array}$ & $\begin{array}{l}\text { A ENTP } \\
\text { demostra } \\
\text { eficácia na } \\
\text { melhora dos } \\
\text { sintomas da BN } \\
\text { de pacientes } \\
\text { com Esclerose } \\
\text { múltipla, } \\
\text { podendo ser um } \\
\text { tratamento } \\
\text { valioso na } \\
\text { sustentação da } \\
\text { melhora clínica. }\end{array}$ \\
\hline
\end{tabular}

G-ENT: grupo eletroestimulação do tibial posterior, GC: grupo controle, G- G-Succinato de Solifenacina: grupo Succinato de Solifenacina, (NI): não informado, (H): homens, (M): mulheres, ENT: eletroestimulação do nervo tibial, FR: frequência, HZ: hertz, us: milissegundos, UU: urgência urinária, IU: incontinência urinária, (NPS): Neuropathic Pain Scale, (ICIQ-SF): International Consultation on Incontinence Questionnaire - Short Form, (OAB-V8): o Overactive Bladder Questionnaire, (OABq): Overactive Bladder Questionnaire, (I-QOI): Incontinence Quality of Life Questionnaire, (OAB-V8): Overactive Bladder Questionnaire. 
Esse atalho permite uma modulação da atividade do músculo detrusor, que leva aos desfechos clínicos encontrados ${ }^{11}$.

A qualidade desses efeitos parece ser mantida mesmo na presença de variados protocolos de intervenção. Isso também pôde ser visto em pacientes com Esclerose múltipla ${ }^{18}$ e em indivíduos com $\mathrm{BN}$ ou bexiga idiopática ${ }^{19}$ submetidos a protocolos de ENTP variados, com parâmetros de 10 ou $20 \mathrm{HZ}$, minutos de intervenção distintos, em sessões diárias ou semanais.

Apesar dos bons resultados, não foram observados efeitos adversos com o uso da eletroestimulação. Entretanto um dos estudos relatou o aparecimento de boca seca em um grupo submetido somente ao fármaco Succinato de Solifenacina ${ }^{16}$. Ainda que possa ser usado para o tratamento da $B N$, esse fármaco pode ter eficácia insuficiente em determinados $\operatorname{casos}^{20}$, além de poder causar efeitos adversos intoleráveis, que podem levar ao abandono do tratamento ${ }^{7,21}$.

A lesão medular foi a patologia mais presente entre os artigos desta revisão. Apesar da maioria dos estudos avaliarem sintomas distintos, foi visto uma melhoria em parâmetros como qualidade de vida, desejo miccional, IU e dor. Dois desses artigos ${ }^{14,15}$, com o mesmo protocolo de intervenção, relataram uma diferença considerável no aumento do limiar do primeiro desejo miccional. Essa desproporção pode ser decorrente do caráter subjetivo desse desfecho, tendo em vista que o primeiro desejo miccional 
depende de vários fatores, por exemplo, do nível de lesão medular dos indivíduos ${ }^{22}$ e da avaliação cognitiva do paciente acerca do problema ${ }^{3}$.

A eletroestimulação também obteve boas repercussões em pacientes com Parkinson e Esclerose múltipla. Nessas disfunções, todos os estudos constataram diminuição da frequência miccional, UU, IU e noctúria. Da mesma forma, a aplicação diária da ENTP de 20 minutos, durante três meses, foi satisfatória ao trazer melhorias à frequência urinária, UU e qualidade de vida de pacientes com Esclerose múltipla ${ }^{23}$. Resultados semelhantes também foram encontrados em um protocolo de 12 meses de eletroestimulação, composto por intervalos sem eletroestimulação ${ }^{17}$.

Além do Parkinson e Esclerose múltipla, a diminuição de sintomas como frequência urinária, noctúria e UU, foi relatada em outras patologias, sendo também observada em indivíduos com AVC, submetidos a 30 minutos de ENTP semanalmente ${ }^{24}$. Com um protocolo equivalente, desfechos semelhantes também foram observados na aplicação de ENTP em doenças como Esclerose múltipla, neoplasias, infecções centrais e Miastenias gravis ${ }^{25}$.

Esta revisão apresentou algumas limitações. Primeiro, dois artigos selecionados foram feitos pelo mesmo autor em um curto período de tempo, o que pode caracterizar um compartilhamento amostral. Segundo os artigos selecionados apresentaram diferentes formas de mensuração dos desfechos, e isso pode se correlacionar com dificuldades na avaliação dos dados. Ainda assim, a ENTP 
demostrou uma margem satisfatória nos seus resultados, além de apresentar desfechos equivalentes nos mesmos sintomas, independente da forma de avaliação.

\section{CONCLUSÃO}

O uso da ENTP apresenta melhorias clínicas significativas no tratamento dos sintomas e na qualidade de vida de pacientes neurológicos com BN, estando ligado com eventos como a diminuição da frequência miccional, IU e UU. Além disso, a ENTP é um tratamento que possui facilidade no seu manejo e não ocasiona efeitos colaterais graves. Portanto, a aplicação dessa intervenção deve ser implementada, com segurança, no tratamento de diferentes doenças neurológicas que causam a BN.

Para o aprimoramento dos efeitos clínicos, ainda se faz necessário o desenvolvimento de novos estudos que investiguem a associação da ENTP com outros tratamentos, como o treinamento dos músculos do assoalho pélvico, tendo em vista os benefícios dessa técnica para portadores de BN.

\section{REFERÊNCIAS}

1.Phé V, Chartier-kastler E, Panicker J. Management of neurogenic bladder in patients with multiple sclerosis. Nat Rev Urol 2016;13:27588. https://doi.org/10.1038/nrurol.2016.53

2.Przydacz M, Denys $P$, Corcos J. What do we know about neurogenic bladder prevalence and management in developing countries and emerging regions of the world? Ann Phys Rehabil Med 2017;60:341-6. https://doi.org/10.1016/j.rehab.2017.02.008

3.Liao L. Evaluation and management of neurogenic bladder: what is new in china? Int J Mol Sci 2015;16:18580-600. https://doi.org/10.3390/ijms160818580

4.Ruffion A, Castro-Diaz D, Patel H, Khalaf K, Onyenwenyi A, Globe D, et al. Systematic review of the epidemiology of urinary incontinence and detrusor overactivity among patients with neurogenic overactive 
bladder.

https://doi.org/10.1159/000353274

5. Flack C, Powell CR. The worldwide economic impact of neurogenic bladder. Curr Bladder Dysfunct Rep 2015;10:350-4. https://doi.org/10.1007/s11884-015-0323-6

6.Zamora IDP, Atiemo HO. Understanding the economic impact of neurogenic lower urinary tract dysfunction. Urol Clin North Am 2017;44:333-43. https://doi.org/10.1016/j.ucl.2017.04.001

7.Starkman J, Smith CP, Staskin DR. Surgical options for drugrefractory overactive bladder patients. Rev Urol 2010;12:97-110. https://www.ncbi.nlm.nih.gov/pmc/articles/PMC2931287/

8. Kabay S, Kabay SC, Cetiner M, Mestan E, Sevim M, Ayas S, et al. The clinical and urodynamic results of percutaneous posterior tibial nerve stimulation on neurogenic detrusor overactivity in patients with parkinson's disease. Urology 2016;87:76-81. https://doi.org/10.1016/j.urology.2015.09.026

9. Perissinotto MC, Ancona CAL, Lucio A, Campos RM, Abreu A. Transcutaneous tibial nerve stimulation in the treatment of lower urinary tract symptoms and its impact on health-related quality of life in patients with parkinson disease. J Wound Ostomy Continence Nurs 2015;42:94-9.

http://repositorio.unicamp.br/jspui/handle/REPOSIP/85388

10.Amarenco G, Ismael SS, Even-schneider A, Raibaut P, Demaillewlodyka S, Parratte $B$, et al. Urodynamic effect of acute transcutaneous posterior tibial nerve stimulation in overactive bladder. Urology 2003; 169:2210-5.

https://doi.org/10.1097/01.ju.0000067446.17576.bd

11. Coolen RL, Groen J, Blok BFM. Electrical stimulation in the treatment of bladder dysfunction: technology update. Med Devices (Auckl) 2019;12:337-45. https://doi.org/10.2147/MDER.S179898

12. Kabay S, Kabay SC, Yucel M, Ozden H, Yilmaz Z, Aras O, et al. The clinical and urodynamic results of a 3-month percutaneous posterior tibial nerve stimulation treatment in patients with multiple sclerosisrelated neurogenic bladder dysfunction. Neurourol Urodyn 2009;28:964-8. https://doi.org/10.1002/nau.20733

13. Mendes KDS, Silveira RCCP, Galvao CM. Revisão integrativa: método de pesquisa para a incorporação de evidências na saúde e na enfermagem. Texto contexto enferm 2008;17:758-64. https://doi.org/10.1590/S0104-07072008000400018

14.Stampas A, Gustafson K, Korupolu R, Smith C, Zhu L, Li S. Bladder neuromodulation in acute spinal cord injury via transcutaneous tibial nerve stimulation: cystometrogram and autonomic nervous system evidence from a randomized control pilot trial. Front Neurosci 2019;13:119. https://doi.org/10.3389/fnins.2019.00119

15.Stampas A, Korupolu R, Zhu L, Smith C, Gustafson K. Safety, feasibility, and efficacy of transcutaneous tibial nerve stimulation in acute spinal cord injury neurogenic. Neuromodulation 2018;22:71622. https://doi.org/10.1111/ner.12855 
16. Chen $\mathrm{G}$, Liao $\mathrm{L}$, Li Y. The possible role of percutaneous tibial nerve stimulation using adhesive skin surface electrodes in patients with neurogenic detrusor overactivity secondary to spinal cord injury. Int Urol Nephrol 2015;47:451-5. https://doi.org/10.1007/s11255-0150911-6

17.Kabay SC, Kabay S, Mestan E, Cetiner M, Ayas S, Sevim M, et al. Long term sustained therapeutic effects of percutaneous posterior tibial nerve stimulation treatment of neurogenic overactive bladder in multiple sclerosis patients: 12-months results. Neurourol Urodyn 2017;36:104-10. https://doi.org/10.1002/nau.22868

18.Zecca C, Panicari L, Disanto G, Maino P, Singh A, Digesu GA, et al. Posterior tibial nerve stimulation in the management of lower urinary tract symptoms in patients with multiple sclerosis. Int Urogynecol J 2015;27:521-7. https://doi.org/10.1007/s00192-015-2814-6

19.Booth J, Connelly L, Dickson S, Duncan F, Lawrence M. The effectiveness of transcutaneous tibial nerve stimulation (TTNS) for adults with overactive bladder syndrome: a systematic review. Neurourol Urodyn

2017;37:528-41. https://doi.org/10.1002/nau.23351

20.Krebs J, Pannek J. Effects of solifenacin in patients with neurogenic detrusor overactivity as a result of spinal cord lesion. Spinal Cord 2013;51:306-9. https://doi.org/10.1038/sc.2012.164

21.Basra $R$, Kelleher C. A review of solifenacin in the treatment of urinary incontinence. Ther Clin Risk Manag 2008;4:117-28. https://doi.org/10.2147/TCRM.S1274

22. Weld KJ, Dmochowski RR. Association of level of injury and bladder behavior in patients with post-traumatic spinal cord injury. Urology 2000;55:490-4. https://doi.org/10.1016/S0090-4295(99)00553-1

23. Sèze $M$, Raibaut $P$, Gallien $P$, Even-Schneider $A$, Denys $P$, Bonniaud $V$, et al. Transcutaneous posterior tibial nerve stimulation for treatment of the overactive bladder syndrome in multiple sclerosis: results of a multicenter prospective study. Neurourol Urodyn 2011;30:306-11. https://doi.org/10.1002/nau.20958

24. Monteiro ÉS, Carvalho LBC, Fukujima MM, Lora MI, Prado GF. Electrical Stimulation of the Posterior Tibialis Nerve Improves Symptoms of Poststroke Neurogenic Overactive Bladder in Men: a randomized controlled trial. Urology 2015;84:509-14. https://doi.org/10.1016/j.urology.2014.05.031

25.Valles-Antuña C, Pérez-Haro ML, González-Ruiz LC, Quintás-Blanco A, Tamargo-Diaz EM, García-Rodríguez J, et al. Estimulación transcutánea del nervio tibial posterior en el tratamiento de la incontinencia urinaria de urgencia refractaria, de origen idiopático y neurogênico. Actas Urol Esp 2017;41:465-70. http://dx.doi.org/10.1016/j.acuro.2017.01.009 